\title{
A Relativistic Complex Optical Potential Calculation for Electron-Beryllium Scattering: Recommended Cross Sections
}

Cite as: J. Phys. Chem. Ref. Data 47, 033103 (2018); https://doi.org/10.1063/1.5047139

Submitted: 04 July 2018 . Accepted: 21 August 2018. Published Online: 12 September 2018

R. P. McEachran, F. Blanco, G. García, and M. J. Brunger

\section{COLLECTIONS}

This paper was selected as Featured
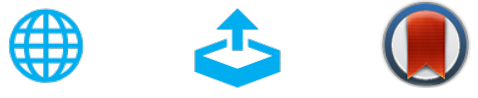

\section{ARTICLES YOU MAY BE INTERESTED IN}

Integral Cross Sections for Electron-Magnesium Scattering Over a Broad Energy Range (0$5000 \mathrm{eV}$ )

Journal of Physical and Chemical Reference Data 47, 043104 (2018); https://

doi.org/10.1063/1.5081132

Reference Values and Reference Correlations for the Thermal Conductivity and Viscosity of Fluids

Journal of Physical and Chemical Reference Data 47, 021501 (2018); https://

doi.org/10.1063/1.5036625

Reference Correlation of the Viscosity of $\mathrm{n}$-Hexadecane from the Triple Point to $673 \mathrm{~K}$ and up to $425 \mathrm{MPa}$

Journal of Physical and Chemical Reference Data 47, 033102 (2018); https://

doi.org/10.1063/1.5039595

\section{Where in the world is AIP Publishing?}

Find out where we are exhibiting next 


\title{
A Relativistic Complex Optical Potential Calculation for Electron-Beryllium Scattering: Recommended Cross Sections
}

\author{
R. P. McEachran \\ Plasma Research Laboratory, Research School of Physics and Engineering, Australian National University, Canberra, \\ Australian Capital Territory 0200, Australia \\ F. Blanco \\ Departamento de Física Atómica, Molecular y Nuclear, Universidad Complutense de Madrid, Avenida Complutense, \\ E-28040 Madrid, Spain \\ G. García \\ Instituto de Física Fundamental, CSIC, Serrano 113-bis, E-28006 Madrid, Spain \\ M. J. Brunger ${ }^{a)}$ \\ College of Science and Engineering, Flinders University, GPO Box 2100, Adelaide, South Australia 5042, Australia
}

(Received 4 July 2018; accepted 21 August 2018; published online 12 September 2018)

\begin{abstract}
We report results from the application of the relativistic complex optical potential (ROP) method to electron-beryllium scattering. The energy range of this study was $0-5000 \mathrm{eV}$, with the results for the integral elastic cross sections, momentum transfer cross sections, summed discrete electronic-state excitation integral cross sections, and total ionisation cross sections (TICSs) being reported. However we will largely focus our discussion here on the TICS, due to its importance in simulating the plasma action on beryllium $(\mathrm{Be})$ in the international thermonuclear reactor. The current level of agreement between the various theoretical approaches to calculating the TICS is well summarised in the work of Maihom et al. [Eur. Phys. J. D 67, 2 (2013)] and Blanco et al. [Plasma Sources Sci. Technol. 26, 085004 (2017)], with the level of accord between them being quite marginal. As a consequence, we revisit this problem with improved scattering potentials over those employed in the work of Blanco et al. In addition, we present results from an application of the binary-encounter-Bethe theory for the electron-Be TICS. We find a quite significant improvement in the level of agreement between the TICS from our new ROP calculation and the earlier B-spline R-matrix and convergent close coupling results [O. Zatsarinny et al., J. Phys. B: At., Mol. Opt. Phys. 49, 235701 (2016)], compared to that reported in the work of Blanco et al. As a result of this improved level of accord, we propose here a recommended TICS for e + Be scattering, as well as for the elastic integral and summed electronic-state excitation cross sections, which also incorporates uncertainty estimates for their validity. Published by AIP Publishing on behalf of the National Institute of Standards and Technology. https://doi.org/10.1063/1.5047139
\end{abstract}

Key words: beryllium; electron scattering cross sections; recommended cross sections.

\section{CONTENTS}

1. Introduction

2

\footnotetext{
a) Author to whom correspondence should be addressed: michael.brunger@ flinders.edu.au

Published by AIP Publishing on behalf of the National Institute of Standards and Technology.
}

2. Theoretical Details ............ 3

2.1. ROP calculation ............. 3

2.2. BEB calculation ............. 3

3. Uncertainty Estimates ............ 4

4. Results and Discussion ............. 4

5. Conclusions ................. 11

Acknowledgments . . . . . . . . . . . . . . . . . . . . . . 12

6. References................... 12 


\section{List of Tables}

1. A selection of the present theoretical ROP results $\left(\times 10^{-16} \mathrm{~cm}^{2}\right)$ for electron scattering from Be. . .

2. Our recommended elastic ICSs, summed electronic-state excitation ICSs, and the TICSs for electron-Be scattering (all in units of $10^{-16} \mathrm{~cm}^{2}$ ) on a fine energy grid. . . . . . . . . .

\section{Introduction}

It is now well known that beryllium (Be) will be one of the materials exposed to the plasma in the international thermonuclear experimental reactor (ITER). ${ }^{1}$ This exposure will lead to the formation of gas-phase $\mathrm{Be}$, with its presence in the fusion edge and divertor plasmas influencing their behaviour due, amongst others, to electron collision processes. ${ }^{2}$ Those processes are quantified by their relevant electron scattering cross sections, in particular, the total ionisation cross section (TICS), which therefore become crucial inputs when attempting to simulate the effect the plasma might have on the materials that are exposed to it.

As a consequence of the above, there has been significant theoretical activity in attempting to calculate cross sections for electron scattering from Be. Focussing on the TICS here, we note plane wave Born approximation (PWBA) results that can be obtained from the method in the work of Deutcsh et al., ${ }^{3,4}$ a distorted-wave with electron scattering (DWIS(N-1)) computation from the work of Bartlett and Stelbovics, ${ }^{5}$ a Deutsch-Märk (DM) calculation from the work of Maihom et al., ${ }^{2}$ the close coupling (CC) and R-matrix with pseudo-states (RMPS) results from the work of Zakrzewski and Ortiz, ${ }^{6}$ a later convergent close coupling (CCC) and a new B-spline R-matrix (BSR) calculation from the work of Zatsarinny et al. ${ }^{7}$ and our own optical potential (OP) computation (Blanco et al.). ${ }^{8}$ Note that as Be is not a particularly easy material to work with, there are no experimental cross sections currently available in the literature for it. All the earlier theoretical TICS ${ }^{3-8}$ are now plotted in Fig. 1, so as to give the reader a clear picture of the extent of the agreement between them. It is apparent from Fig. 1 that while some of the theoretical results are in quite good agreement with some of the others, in general there is a rather wide divergence in the reported absolute values for the TICS. For example, at the energy where a maximum in the cross section is found, the variance in the magnitude of that TICS can be as much as a factor of two. Therefore we have decided to revisit this scattering system, using our relativistic complex optical potential (ROP) method $^{9}$ and with improved scattering potentials (see later) over those employed in the work of Blanco et al. ${ }^{8}$ in order to try and further clarify this situation. Furthermore, as is evident from Fig. 1, many of the available theories only go up to $100 \mathrm{eV}$ incident electron energy. To be useful in modeling applications, particularly with respect to high-temperature plasmas, a much larger energy range is required. Therefore, another rationale for the present ROP calculation is to hopefully enable us to extend the cross

\section{List of Figures}

1. Present status for theoretical electron-Be TICS

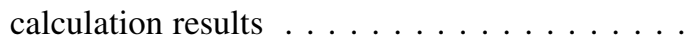

2. TICSs for electron-Be scattering, including our new ROP and BEB results . . . . . . . . . . .

3. Summary plot of our recommended cross section data $\left(\times 10^{-16} \mathrm{~cm}^{2}\right)$ for $e^{-}-$Be scattering $\ldots \ldots$

section data to those higher energies. In addition to our ROP computation, we also report new binary encounter Bethe (BEB) calculation results, as there is a large body of data that suggest that this quite simple approach can provide remarkably accurate ionisation cross sections. ${ }^{10}$ Finally we will present a recommended TICS for electron-Be scattering, with uncertainty limits, over an extended energy range for use in simulation studies. Recommended data for elastic scattering and the summed electronic-state excitation cross section, with associated confidence limits, will also be provided.

The structure for the remainder of this short paper is as follows. In Sec. 2, we describe our ROP and BEB calculations, while in Sec. 3, we discuss the uncertainty estimates we quote on our recommended data. The present computational results and a discussion of these results are given in Sec. 4. Also included in Sec. 4 will be our recommended elastic integral cross section (ICS), summed electronic-state excitation ICS, and TICS values for this scattering system, with uncertainty estimates on those cross sections additionally being provided. Finally, in Sec. 5, some conclusions from the present investigation will be given.

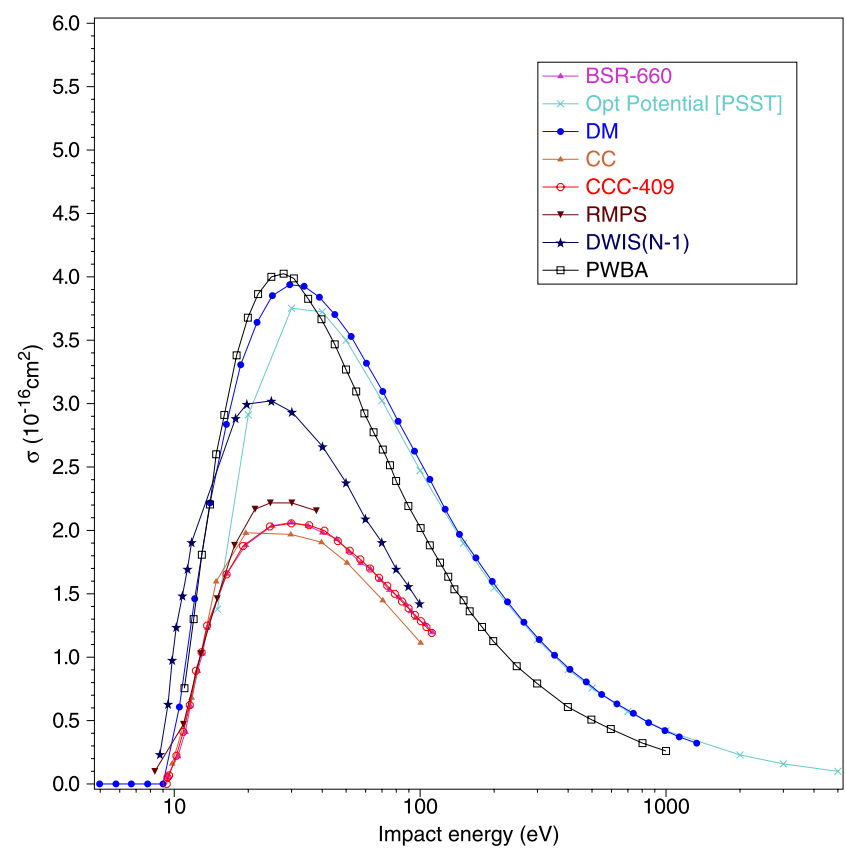

FIG. 1. Present status for theoretical electron-Be TICS calculation results. See the legend for further details. 


\section{Theoretical Details}

\subsection{ROP calculation}

In this work, the elastic and absorption cross sections were calculated using a ROP method. This method was originally developed by Chen et al. ${ }^{9}$ and used to calculate total and differential cross sections as well as spin polarisation parameters for relatively low energy electrons and positrons incident upon krypton. That paper will be referred to as I hereafter. It has subsequently been successfully used in several different fields of atomic scattering, in particular, in the analysis of energy-loss spectra at several keV by Vos et al., ${ }^{11}$ in the investigation of the behaviour of the Sherman function in electron scattering from xenon, ${ }^{12}$ for low energy positron interactions with krypton, ${ }^{13}$ and for positronium formation in the noble gases. ${ }^{14}$

The ROP method is based upon the solution of the Dirac scattering equations. Here the radial integral equations for the large and small components of the scattering wavefunctions, $F_{0}(x)$ and $G_{0}(x)$, can be expressed in matrix form as

$$
\begin{aligned}
\left(\begin{array}{l}
F_{0}(x) \\
G_{0}(x)
\end{array}\right)= & \left(\begin{array}{l}
v_{1}\left(k_{0} x\right) \\
v_{2}\left(k_{0} x\right)
\end{array}\right)+\frac{1}{k_{0}} \int_{0}^{x} d r G_{\Gamma_{0}}^{P}(x, r) \\
& \times\left[U(r)\left(\begin{array}{l}
F_{0}(r) \\
G_{0}(r)
\end{array}\right)-\left(\begin{array}{l}
\bar{W}_{P}\left(\kappa_{2} ; r\right) \\
\bar{W}_{Q}\left(\kappa_{2} ; r\right)
\end{array}\right)\right. \\
& \left.-i U_{\mathrm{abs}}(r)\left(\begin{array}{l}
F_{0}(r) \\
G_{0}(r)
\end{array}\right)\right] .
\end{aligned}
$$

In Eq. (1), the local potential $U(r)$ is the sum of the static and polarization potentials, while $\bar{W}_{P}\left(\kappa_{2} ; r\right)$ and $\bar{W}_{Q}\left(\kappa_{2} ; r\right)$ are the large and small components of the non-local exchange terms. Finally, $U_{\mathrm{abs}}(r)$ is the non-local absorption potential and is determined as an expansion over the inelastic channels of the target atom. These inelastic channels include both excitation of the higher lying bound states and single ionisation of the target as given by Eq. (21b) of I. The above Green's function $G_{\Gamma_{0}}^{P}(x, r)$ can be expressed in terms of Riccati-Bessel and Riccati-Neumann functions [see Eq. (23) of I for details]. The angular momentum quantum number $\kappa_{2}= \pm 1, \pm 2, \ldots$ of the incident electron can be defined in terms of its orbital angular quantum number $l_{2}$ and the total angular momentum quantum number $j_{2}$ according to $j_{2}=\left|\kappa_{2}\right|-\frac{1}{2}$ with $l_{2}=\kappa_{2}$ if $\kappa_{2}>0$ and $l_{2}=-\kappa_{2}-1$ if $\kappa_{2}<0$.

In particular, the polarization potential was determined by the polarized-orbital method ${ }^{15,16}$ and included the first 7 multipole potentials plus the corresponding dynamic polarization potential. ${ }^{17}$ Thus, asymptotically the polarization potential contained all terms up to and including those corresponding to $r^{-14}$

The ground state wavefunction of beryllium was determined in a single configuration calculation using the multiconfiguration Dirac-Fock (MCDF) programme of Grant et al. ${ }^{18}$ This wavefunction was used in the calculation of the static potential as well as the bound and continuum state coupling potentials in the absorption potential. For the excited bound states of beryllium, which were used in the absorption potential, we included those 10 states where one of the electrons in the outer $2 \mathrm{~s}$ valence shell was excited to a higher lying $n \mathrm{p}^{1,3} \mathrm{P}$ state with $n=2-6$ inclusive. For the case of ionisation, we included those continuum states which correspond to an orbital angular momentum of $0-4$; this gives rise to up to 49 ionisation channels depending on the total angular momentum of the incident electron.

Equation (1) was solved iteratively to obtain the complex phase shifts $\eta_{l_{2}}^{ \pm}=\delta_{l_{2}}^{ \pm}+i \gamma_{l_{2}}^{ \pm}$with $\gamma_{l_{2}}^{ \pm} \geq 0$. Here the (+) sign corresponds to "spin-up" $\left(j_{2}=l_{2}+\frac{1}{2}\right)$ and the (-) sign corresponds to "spin-down" $\left(j_{2}=l_{2}-\frac{1}{2}\right)$. In terms of these phase shifts, the elastic cross section is given by

$$
\begin{aligned}
\sigma^{\mathrm{el}}\left(k^{2}\right)= & \frac{2 \pi}{k^{2}} \sum_{l_{2}=0}^{\infty}\left\{\left(l_{2}+1\right) \exp \left(-2 \gamma_{l_{2}}^{+}\right)\left[\cosh 2 \gamma_{l_{2}}^{+}-\cos 2 \delta_{l_{2}}^{+}\right]\right. \\
& \left.+l_{2} \exp \left(-2 \gamma_{l_{2}}^{-}\right)\left[\cosh 2 \gamma_{l_{2}}^{-}-\cos 2 \delta_{l_{2}}^{-}\right]\right\}
\end{aligned}
$$

while the absorption cross section is given by

$$
\begin{aligned}
\sigma^{\mathrm{abs}}\left(k^{2}\right) & =\frac{\pi}{k^{2}} \sum_{l_{2}=0}\left\{\left(l_{2}+1\right)\left[1-\exp \left(-4 \gamma_{l_{2}}^{+}\right)\right]\right. \\
& \left.+l_{2}\left[1-\exp \left(-4 \gamma_{l_{2}}^{-},\right)\right]\right\}
\end{aligned}
$$

Here $k$ is the relativistic wavenumber of the incident electron.

\subsection{BEB calculation}

The BEB approach for atomic systems is well described in the recent review of Tanaka et al., ${ }^{10}$ and so we do not go into detail here. Rather we note that the ionisation cross section for a particular orbital $\left(2 s^{2}\right.$ here for Be $)$ is given by

$$
\sigma_{\mathrm{BEB}}(t)=\frac{S}{t+u+1}\left[\frac{\ln (t)}{2}\left(1-\frac{1}{t^{2}}\right)+1-\frac{1}{t}-\frac{\ln (t)}{t+1}\right],
$$

where $t=T / B, u=U / B, S=4 \pi a_{0}^{2} N R^{2} / B^{2}, a_{0}$ is the Bohr radius $(0.529 \AA), R$ is the Rydberg energy $(13.6057 \mathrm{eV})$, and $T$ is the incident electron energy. $N, B$, and $U$ are the electron occupation number, the binding energy (ionisation potential $=9.3227 \mathrm{eV}^{19}$ ), and the average kinetic energy of the orbital, respectively. In this case, we have used the experimental value of the ionisation potential (from the ground $2 \mathrm{~s}^{2}$ state) for $B$, while $N$ and $U$ are determined from a DFT computation at the B3LYP level with GAUSSIAN. ${ }^{20}$ The present BEB TICS is plotted in Fig. 2. Note that there was an earlier BEB electron-Be TICS from the work of Maihom et al., ${ }^{2}$ with a Hartree-Fock model chemistry. The BEB result in the work of Maihom et al. differs from the present, largely in terms of the magnitude of the TICS at its maximum value (they are $\sim 20 \%$ higher in value), by more than one would anticipate simply on the basis of the different model chemistries used in the two calculations. We have checked our BEB result very carefully and believe it is correct. As a consequence, the earlier result ${ }^{2}$ has not been included in either Fig. 1 or Fig. 2. 


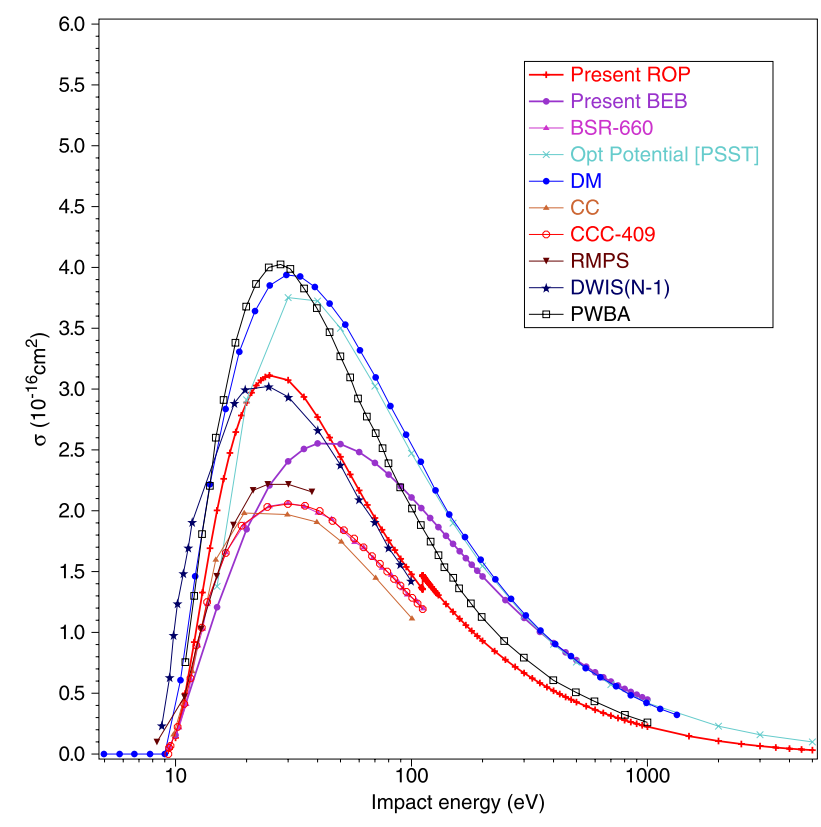

FIG. 2. TICSs for electron-Be scattering, including our new ROP and BEB results. See the legend for further details.

\section{Uncertainty Estimates}

It is very important for the modeling community to have uncertainty limits on the recommended cross section data, when they come to apply those cross sections to the application of interest. ${ }^{21}$ In this case, we are proposing recommended data from theory alone, and given that all the theories have been properly applied and are converged their intrinsic uncertainties are probably $1 \%$ or less. Under these circumstances, we have adopted as our uncertainties the confidence limits that are typically associated with benchmark experiments, if such experiments were in fact possible for electronBe scattering. Those experimental uncertainties are detailed in the work of Buckman et al. ${ }^{22}$ and typically are $\pm 10 \%$ for elastic scattering, $\pm 30 \%$ for electronic-state excitation, and $\pm 5 \%$ for the TICS. In the present application, however, we have formed the confidence limits on the TICS from the standard deviation on the average values, at each energy, of the six theoretical results [CC, CCC, BSR, RMPS, DWIS(N-1), and ROP] we employed to determine the recommended ionisation data below $100 \mathrm{eV}$ (see later). This approach led to a conservative $\pm 20 \%$ uncertainty on our recommended TICS. Note that while that estimate is conservative, it is not unreasonable. Recent TICS measurements with the primary alcohols, ${ }^{23-25}$ which are liquids at room temperature, reported uncertainties up to $\sim 12 \%$. As any measurements with beryllium would be a degree of difficulty more challenging, our $\pm 20 \%$ uncertainty estimate on our recommended TICS is highly plausible.

\section{Results and Discussion}

In Table 1, we present a summary of all the present ROP calculation results for electron-Be scattering. These consist of data for the elastic ICS, for energies in the range $0-5000 \mathrm{eV}$, the momentum transfer cross section (MTCS), again for energies between 0 and $5000 \mathrm{eV}$, the summed electronic-state excitation cross sections, for energies from threshold $(2.8 \mathrm{eV})$ to $5000 \mathrm{eV}$, and the TICS, for energies from its threshold $(\sim 10 \mathrm{eV})$ to $5000 \mathrm{eV}$. Note that we do not discuss our MTCS results further, but they are included in Table 1 because in some modeling applications they are important parameters. One important example of this is in simulating electron transport under an applied external electric field through the background gas of interest (i.e., swarm physics). ${ }^{26-29}$ While we do not explicitly include plots of the available theoretical ICS results for either elastic scattering ${ }^{7,8}$ or the summed electronic-states, ${ }^{7,8,30}$ it would be remiss of us not to provide a brief summary of their current status.

For the elastic ICS, the BSR and CCC results were found to be in good agreement over their common energy range $(<10 \%$ difference) and, for energies above about $2 \mathrm{eV}$ (to $\sim 10 \%$ or better) and up to $100 \mathrm{eV}$, were also found to be in good agreement with the optical model result from the work of Blanco et al. ${ }^{8}$ This good level of accord between the sophisticated BSR method and the OP model approach, in the elastic channel, is by no means unique, having been also seen earlier for electron-atomic iodine scattering. ${ }^{31}$ The present ROP elastic ICS reinforces this scenario, being in very good agreement with the BSR result over their entire common energy range $(0-100 \mathrm{eV})$ to typically better than $20 \%$ [with the worst agreement being at the lowest common energies $(\sim 0.01 \mathrm{eV}-0.2 \mathrm{eV})]$. As a consequence of this level of accord, we can construct a recommended elastic $e^{-}+\mathrm{Be}$ scattering cross section that is composed of the BSR result for energies between 0 and $100 \mathrm{eV}$ and our current ROP result for energies between 100 and $5000 \mathrm{eV}$. A selection of these recommended elastic ICS results is given in Table 2 with the estimated uncertainty on them being $\pm 10 \%$.

The situation with respect to the sum of electronic-excited states is, however, much less clear. While the BSR and CCC results are typically again in good accord, to better than $10 \%$, from threshold to $100 \mathrm{eV}$, the OP result of Blanco et al. ${ }^{8}$ is very different from them both. While we would anticipate a divergence between them near-threshold where the OP model, with a local-exchange potential to describe exchange scattering and a standard description for the polarisation potential, is known to be less accurate, ${ }^{8}$ the factor of 4 difference at $100 \mathrm{eV}$ was highly surprising and indeed formed an important rationale for the present ROP application. When we compare our current summed electronic-state excitation ROP ICS to the earlier results, ${ }^{7,8}$ we find fair agreement, across most of the common energy range from threshold $-100 \mathrm{eV}$, with the $\mathrm{CCC}$ and BSR calculations. The only exception to this general claim is nearthreshold, where our $2 \mathrm{~s}^{2} \rightarrow 2 \mathrm{~s} 2 \mathrm{p} 2^{3} \mathrm{P}$ excitation is apparently much smaller than the corresponding BSR cross section.

The excitation of the $2^{3} \mathrm{P}$ state in $\mathrm{Be}$, under a LS-coupling scheme, would be via the exchange interaction, and this would normally be expected to be much smaller in magnitude than the dipole-allowed $2^{1} \mathrm{P}$ excitation. However, here there are some strong near-threshold resonance states, ${ }^{32}$ found in both the CCC and BSR computations, which greatly enhance 
TABLE 1. A selection of the present theoretical ROP results $\left(\times 10^{-16} \mathrm{~cm}^{2}\right)$ for electron scattering from Be. Here the acronym MTCS denotes the momentum transfer cross section

\begin{tabular}{|c|c|c|c|c|}
\hline Energy $(\mathrm{eV})$ & Elastic $\left(\times 10^{-16} \mathrm{~cm}^{2}\right)$ & Inelastic $\left(\times 10^{-16} \mathrm{~cm}^{2}\right)$ & TICS $\left(\times 10^{-16} \mathrm{~cm}^{2}\right)$ & MTCS $\left(\times 10^{-16} \mathrm{~cm}^{2}\right)$ \\
\hline 0 & 0.005 & 0 & 0 & 0.005 \\
\hline 0.04 & 16.619 & 0 & 0 & 25.252 \\
\hline 0.08 & 44.17 & 0 & 0 & 63.892 \\
\hline 0.12 & 94.666 & 0 & 0 & 126.592 \\
\hline 0.16 & 181.729 & 0 & 0 & 223.249 \\
\hline 0.19 & 272.871 & 0 & 0 & 315.051 \\
\hline 0.22 & 371.223 & 0 & 0 & 404.166 \\
\hline 0.26 & 464.859 & 0 & 0 & 470.959 \\
\hline 0.32 & 467.746 & 0 & 0 & 431.341 \\
\hline 0.36 & 420.658 & 0 & 0 & 367.506 \\
\hline 0.4 & 369.764 & 0 & 0 & 307.931 \\
\hline 0.44 & 325.47 & 0 & 0 & 259.773 \\
\hline 0.48 & 289.403 & 0 & 0 & 222.442 \\
\hline 0.6 & 218.36 & 0 & 0 & 153.378 \\
\hline 0.8 & 161.183 & 0 & 0 & 102.676 \\
\hline 1 & 132.521 & 0 & 0 & 79.569 \\
\hline 1.4 & 102.779 & 0 & 0 & 58.029 \\
\hline 2 & 80.408 & 0 & 0 & 43.894 \\
\hline 2.6 & 67.174 & 0 & 0 & 36.323 \\
\hline 2.9 & 58.459983 & 0.023778 & 0 & 33.975614 \\
\hline 4 & 50.382931 & 0.034919 & 0 & 28.198544 \\
\hline 4.8 & 45.880451 & 0.033316 & 0 & 24.67233 \\
\hline 5.6 & 41.518683 & 1.432638 & 0 & 20.543089 \\
\hline 6 & 39.326243 & 3.557447 & 0 & 17.744882 \\
\hline 6.8 & 35.962291 & 7.095222 & 0 & 13.638445 \\
\hline 7.6 & 33.072483 & 9.864948 & 0 & 10.87313 \\
\hline 8.4 & 30.520351 & 12.009985 & 0 & 8.959686 \\
\hline 9.2 & 28.215034 & 13.860551 & 0 & 7.525776 \\
\hline 13 & 20.52985 & 17.40913 & 1.32778 & 4.18305 \\
\hline 17 & 16.08154 & 17.81907 & 2.47498 & 2.88006 \\
\hline 21 & 13.44764 & 17.79879 & 2.97001 & 2.25224 \\
\hline 25 & 11.65655 & 17.27077 & 3.11284 & 1.90206 \\
\hline 45 & 7.53309 & 14.15983 & 2.60157 & 1.10884 \\
\hline 65 & 5.8253 & 11.84275 & 2.04774 & 0.78744 \\
\hline 85 & 4.8296 & 9.95909 & 1.67695 & 0.60173 \\
\hline 111 & 3.99371 & 8.23668 & 1.35893 & $\ldots$ \\
\hline 111.4 & 3.98333 & 8.21431 & 1.35501 & $\ldots$ \\
\hline 111.5175 & 3.92773 & 8.18855 & 1.46583 & $\ldots$ \\
\hline 112 & 3.9154 & 8.16178 & 1.46184 & $\ldots$ \\
\hline 118 & 3.76908 & 7.84381 & 1.40584 & $\ldots$ \\
\hline 140 & 3.31938 & 6.86087 & 1.23353 & 0.32279 \\
\hline 180 & 2.73724 & 5.68814 & 1.01253 & 0.23373 \\
\hline 250 & 2.10127 & 4.36431 & 0.77566 & 0.14837 \\
\hline 300 & 1.8039 & 3.66582 & 0.66603 & 0.11377 \\
\hline 400 & 1.40685 & 2.70648 & 0.52033 & 0.07363 \\
\hline 500 & 1.15345 & 2.09458 & 0.4271 & 0.052 \\
\hline 700 & 0.8483 & 1.46152 & 0.31567 & 0.03035 \\
\hline 1000 & 0.60772 & 0.92789 & 0.22499 & 0.01711 \\
\hline 3000 & 0.21005 & 0.17381 & 0.06571 & 0.00255 \\
\hline 5000 & 0.12696 & 0.07769 & 0.03231 & 0.00103 \\
\hline
\end{tabular}

the magnitude of the $2^{3} \mathrm{P}$ state excitation cross section. As the present ROP calculation includes only a relatively small number of excited states, it cannot replicate this type of resonance. Therefore, to construct our recommended data set, we prefer the BSR and CCC results in this near-threshold energy regime. However, above the near-threshold region, the level of accord between our ROP and the BSR calculation is typically better than $30 \%$ up to $100 \mathrm{eV}$. As a consequence, we believe we are now in a position to recommend a summed electronic-state excitation ICS for $e^{-}-\mathrm{Be}$ scattering. This recommended ICS is formed from the BSR results from threshold to $70 \mathrm{eV}$, and for $E_{0}=70-1000 \mathrm{eV}$ from the present ROP computation (scaled downwards by a factor of 0.64 at $70 \mathrm{eV}$ to ensure continuity). Note that we have truncated the BSR cross sections at $70 \mathrm{eV}$ due to some convergence issues with their higher energy data for the excited electronic-states. In the energy range $1000-5000 \mathrm{eV}$ we prefer the cross sections from the Livermore computation, ${ }^{30}$ due to their clearly exhibiting the anticipated Born-like high-energy asymptotic behaviour. Values for a selection of our recommended ICS here can also be found in Table 2, with the uncertainty limits of those data now being $\pm 30 \%$. 
TABLE 2. Our recommended elastic ICSs, summed electronic-state excitation ICSs, and the TICSs for electronBe scattering (all in units of $10^{-16} \mathrm{~cm}^{2}$ ) on a fine energy grid. The uncertainties on the elastic ICS are $\sim \pm 10 \%$, the uncertainties on the summed electronic state excitation ICS are $\sim \pm 30 \%$, and the uncertainties on the TICS are $\sim \pm 20 \%$

\begin{tabular}{|c|c|c|c|}
\hline Energy $(\mathrm{eV})$ & Elastic $\left(\times 10^{-16} \mathrm{~cm}^{2}\right)$ & Inelastic $\left(\times 10^{-16} \mathrm{~cm}^{2}\right)$ & TICS $\left(\times 10^{-16} \mathrm{~cm}^{2}\right)$ \\
\hline $2.643080 \times 10^{-4}$ & 3.49832 & & \\
\hline $5.292000 \times 10^{-4}$ & 2.01029 & & \\
\hline $8.026390 \times 10^{-4}$ & 1.63318 & & \\
\hline $1.059571 \times 10^{-3}$ & 1.45515 & & \\
\hline $1.335457 \times 10^{-3}$ & 1.38950 & & \\
\hline $2.673868 \times 10^{-3}$ & 1.35779 & & \\
\hline $4.055459 \times 10^{-3}$ & 1.48913 & & \\
\hline $5.353527 \times 10^{-3}$ & 1.63318 & & \\
\hline $6.747610 \times 10^{-3}$ & 1.83298 & & \\
\hline $8.119887 \times 10^{-3}$ & 2.05723 & & \\
\hline $9.771247 \times 10^{-3}$ & 2.25623 & & \\
\hline $1.071890 \times 10^{-2}$ & 2.47448 & & \\
\hline $1.231544 \times 10^{-2}$ & 2.71384 & & \\
\hline $1.414979 \times 10^{-2}$ & 3.04585 & & \\
\hline $2.704955 \times 10^{-2}$ & 6.37545 & & \\
\hline $4.102702 \times 10^{-2}$ & 11.0946 & & \\
\hline $5.415892 \times 10^{-2}$ & 16.0513 & & \\
\hline $6.826058 \times 10^{-2}$ & 22.6929 & & \\
\hline $8.214290 \times 10^{-2}$ & 30.6351 & & \\
\hline $9.437783 \times 10^{-2}$ & 38.5896 & & \\
\hline 0.108437 & 48.6094 & & \\
\hline 0.118953 & 59.8329 & & \\
\hline 0.136672 & 71.9681 & & \\
\hline 0.164467 & 104.121 & & \\
\hline 0.188965 & 147.204 & & \\
\hline 0.197916 & 173.021 & & \\
\hline 0.217112 & 198.724 & & \\
\hline 0.227397 & 228.244 & & \\
\hline 0.238168 & 250.323 & & \\
\hline 0.249451 & 280.944 & & \\
\hline 0.273644 & 315.319 & & \\
\hline 0.300184 & 345.820 & & \\
\hline 0.329297 & 370.612 & & \\
\hline 0.344896 & 388.124 & & \\
\hline 0.396269 & 362.160 & & \\
\hline 0.434701 & 322.678 & & \\
\hline 0.499450 & 294.219 & & \\
\hline 0.523110 & 268.269 & & \\
\hline 0.690551 & 208.113 & & \\
\hline 0.793409 & 169.071 & & \\
\hline 0.870359 & 147.204 & & \\
\hline 1.09699 & 128.165 & & \\
\hline 1.20338 & 116.861 & & \\
\hline 1.44812 & 104.121 & & \\
\hline 2.52354 & 64.1239 & & \\
\hline 2.76829 & 54.5557 & & \\
\hline 2.85700 & & 5.96674 & \\
\hline 2.99305 & & 11.9040 & \\
\hline 3.12909 & & 15.8116 & \\
\hline 3.26514 & & 16.6499 & \\
\hline 3.33129 & 43.3102 & & \\
\hline 3.40119 & & 15.9836 & \\
\hline 3.53724 & & 15.1212 & \\
\hline 3.67328 & & 14.3035 & \\
\hline 3.80933 & & 13.5462 & \\
\hline 3.94538 & & 12.9194 & \\
\hline 4.08143 & & 12.4178 & \\
\hline 4.19871 & 36.0073 & & \\
\hline 4.21748 & & 11.9512 & \\
\hline 4.35352 & & 11.4474 & \\
\hline 4.48957 & & 10.9136 & \\
\hline 4.62562 & & 10.4048 & \\
\hline 4.76167 & & 9.96516 & \\
\hline
\end{tabular}


TABLE 2. Our recommended elastic ICSs, summed electronic-state excitation ICSs, and the TICSs for electronBe scattering (all in units of $10^{-16} \mathrm{~cm}^{2}$ ) on a fine energy grid. The uncertainties on the elastic ICS are $\sim \pm 10 \%$, the uncertainties on the summed electronic state excitation ICS are $\sim \pm 30 \%$, and the uncertainties on the TICS are $\sim \pm 20 \%$ - Continued

\begin{tabular}{|c|c|c|c|}
\hline$\overline{\text { Energy }(\mathrm{eV})}$ & Elastic $\left(\times 10^{-16} \mathrm{~cm}^{2}\right)$ & Inelastic $\left(\times 10^{-16} \mathrm{~cm}^{2}\right)$ & TICS $\left(\times 10^{-16} \mathrm{~cm}^{2}\right)$ \\
\hline 4.89771 & & 9.60364 & \\
\hline 5.03376 & & 9.30150 & \\
\hline 5.16981 & & 9.04227 & \\
\hline 5.30586 & & 9.21333 & \\
\hline 5.44190 & & 8.80644 & \\
\hline 5.57795 & & 8.56914 & \\
\hline 5.71400 & & 8.40721 & \\
\hline 5.80522 & 28.5851 & & \\
\hline 5.85005 & & 8.30230 & \\
\hline 5.98609 & & 8.27679 & \\
\hline 6.12214 & & 8.45681 & \\
\hline 6.25819 & & 8.44418 & \\
\hline 6.39424 & & 8.33891 & \\
\hline 6.53028 & & 8.45619 & \\
\hline 6.66633 & & 8.50689 & \\
\hline 6.80238 & & 8.51790 & \\
\hline 6.93843 & & 8.67892 & \\
\hline 7.07447 & & 8.87318 & \\
\hline 7.21052 & & 9.12521 & \\
\hline 7.34657 & & 8.98378 & \\
\hline 7.48262 & & 9.13276 & \\
\hline 7.61866 & & 9.32367 & \\
\hline 7.75471 & & 9.40420 & \\
\hline 7.89076 & & 9.63917 & \\
\hline 8.02644 & 23.2226 & & \\
\hline 8.02681 & & 9.66719 & \\
\hline 8.16286 & & 9.93027 & \\
\hline 8.29890 & & 10.1361 & \\
\hline 8.43495 & & 10.2472 & \\
\hline 8.57100 & & 10.6036 & \\
\hline 8.70705 & & 10.6040 & \\
\hline 8.84309 & & 10.7190 & \\
\hline 8.97914 & & 10.8481 & \\
\hline 9.11519 & & 11.0712 & \\
\hline 9.25124 & & 11.0994 & \\
\hline 9.38728 & & 11.1612 & \\
\hline 9.40000 & & & 0.143623 \\
\hline 9.52333 & & 11.3083 & \\
\hline 10.0000 & & & 0.325492 \\
\hline 10.1165 & 20.2190 & & \\
\hline 10.2036 & & 11.7108 & \\
\hline 10.2377 & & & 0.397555 \\
\hline 10.4811 & & & 0.468432 \\
\hline 10.7303 & & & 0.544638 \\
\hline 10.8838 & & 11.7194 & \\
\hline 10.9854 & & & 0.622755 \\
\hline 11.2466 & & & 0.711917 \\
\hline 11.5140 & & & 0.810643 \\
\hline 11.5640 & & 11.6210 & \\
\hline 11.7877 & & & 0.912698 \\
\hline 12.0679 & & & 1.00400 \\
\hline 12.1739 & 16.4263 & & \\
\hline 12.2443 & & 11.5491 & \\
\hline 12.3548 & & & 1.09355 \\
\hline 12.6486 & & & 1.17316 \\
\hline 12.9245 & & 11.4414 & \\
\hline 12.9493 & & & 1.25158 \\
\hline 13.2571 & & & 1.34188 \\
\hline 13.5723 & & & 1.43684 \\
\hline 13.6048 & & 11.3634 & \\
\hline 13.8950 & & & 1.51130 \\
\hline 13.9872 & 14.6356 & & \\
\hline 14.2253 & & & 1.58046 \\
\hline
\end{tabular}


TABLE 2. Our recommended elastic ICSs, summed electronic-state excitation ICSs, and the TICSs for electronBe scattering (all in units of $10^{-16} \mathrm{~cm}^{2}$ ) on a fine energy grid. The uncertainties on the elastic ICS are $\sim \pm 10 \%$, the uncertainties on the summed electronic state excitation ICS are $\sim \pm 30 \%$, and the uncertainties on the TICS are $\sim \pm 20 \%$ - Continued

\begin{tabular}{|c|c|c|c|}
\hline$\overline{\text { Energy }(\mathrm{eV})}$ & Elastic $\left(\times 10^{-16} \mathrm{~cm}^{2}\right)$ & Inelastic $\left(\times 10^{-16} \mathrm{~cm}^{2}\right)$ & TICS $\left(\times 10^{-16} \mathrm{~cm}^{2}\right)$ \\
\hline 14.5635 & & & 1.65032 \\
\hline 14.9097 & & & 1.71808 \\
\hline 15.2642 & & & 1.77600 \\
\hline 15.6271 & & & 1.83450 \\
\hline 15.9986 & & & 1.89439 \\
\hline 16.3257 & & 10.9977 & \\
\hline 16.3789 & & & 1.95105 \\
\hline 16.7683 & & & 1.99523 \\
\hline 17.1670 & & & 2.03932 \\
\hline 17.5751 & & & 2.08284 \\
\hline 17.9929 & & & 2.12328 \\
\hline 18.4207 & & & 2.15880 \\
\hline 18.4642 & 10.8415 & & \\
\hline 18.8586 & & & 2.19512 \\
\hline 19.0467 & & 10.7680 & \\
\hline 19.3070 & & & 2.22843 \\
\hline 19.7660 & & & 2.25383 \\
\hline 20.2359 & & & 2.26797 \\
\hline 20.7170 & & & 2.28144 \\
\hline 21.2095 & & & 2.29449 \\
\hline 21.7137 & & & 2.30683 \\
\hline 22.2300 & & & 2.31879 \\
\hline 22.7585 & & & 2.33017 \\
\hline 23.2995 & & & 2.34108 \\
\hline 23.8534 & & & 2.35165 \\
\hline 24.3742 & 8.80769 & & \\
\hline 24.4205 & & & 2.36150 \\
\hline 24.4886 & & 10.2224 & \\
\hline 25.0011 & & & 2.36861 \\
\hline 25.5955 & & & 2.36782 \\
\hline 26.2040 & & & 2.36674 \\
\hline 26.8270 & & & 2.36565 \\
\hline 27.4647 & & & 2.36452 \\
\hline 28.1177 & & & 2.36337 \\
\hline 28.7862 & & & 2.36219 \\
\hline 29.4705 & & & 2.36099 \\
\hline 29.9305 & & 9.78473 & \\
\hline 30.1711 & & & 2.35763 \\
\hline 30.7213 & 7.15545 & & \\
\hline 30.8884 & & & 2.34874 \\
\hline 31.6228 & & & 2.33964 \\
\hline 32.3746 & & & 2.33033 \\
\hline 33.1442 & & & 2.32079 \\
\hline 33.9322 & & & 2.31102 \\
\hline 34.7389 & & & 2.30103 \\
\hline 35.3724 & & 9.26335 & \\
\hline 35.5648 & & & 2.28920 \\
\hline 36.4103 & & & 2.27577 \\
\hline 37.2759 & & & 2.26202 \\
\hline 38.1621 & & & 2.24795 \\
\hline 39.0694 & & & 2.23353 \\
\hline 39.9982 & & & 2.21834 \\
\hline 40.5546 & 6.22997 & & \\
\hline 40.8143 & & 8.95107 & \\
\hline 40.9491 & & & 2.20185 \\
\hline 41.9227 & & & 2.18399 \\
\hline 42.9193 & & & 2.16570 \\
\hline 43.9397 & & & 2.14697 \\
\hline 44.9843 & & & 2.12780 \\
\hline 46.0538 & & & 2.10785 \\
\hline 46.2562 & & 8.58205 & \\
\hline 46.5951 & 5.30043 & & \\
\hline 47.1487 & & & 2.08492 \\
\hline
\end{tabular}


TABLE 2. Our recommended elastic ICSs, summed electronic-state excitation ICSs, and the TICSs for electronBe scattering (all in units of $10^{-16} \mathrm{~cm}^{2}$ ) on a fine energy grid. The uncertainties on the elastic ICS are $\sim \pm 10 \%$, the uncertainties on the summed electronic state excitation ICS are $\sim \pm 30 \%$, and the uncertainties on the TICS are $\sim \pm 20 \%$ - Continued

\begin{tabular}{|c|c|c|c|}
\hline$\overline{\text { Energy }(\mathrm{eV})}$ & Elastic $\left(\times 10^{-16} \mathrm{~cm}^{2}\right)$ & Inelastic $\left(\times 10^{-16} \mathrm{~cm}^{2}\right)$ & TICS $\left(\times 10^{-16} \mathrm{~cm}^{2}\right)$ \\
\hline 48.2696 & & & 2.06148 \\
\hline 49.4171 & & & 2.03748 \\
\hline 50.5920 & & & 2.01322 \\
\hline 51.6981 & & 8.12204 & \\
\hline 51.7947 & & & 1.98922 \\
\hline 53.0261 & & & 1.96554 \\
\hline 54.2868 & & & 1.94131 \\
\hline 55.5774 & & & 1.91674 \\
\hline 56.8987 & & & 1.89191 \\
\hline 57.1400 & & 7.85874 & \\
\hline 58.2514 & & & 1.86847 \\
\hline 58.7273 & 4.50957 & & \\
\hline 59.6362 & & & 1.84547 \\
\hline 61.0540 & & & 1.82424 \\
\hline 62.5055 & & & 1.80313 \\
\hline 62.5819 & & 7.64566 & \\
\hline 63.9915 & & & 1.77881 \\
\hline 65.5128 & & & 1.75370 \\
\hline 67.0704 & & & 1.72839 \\
\hline 68.0238 & & 7.37533 & \\
\hline 68.6649 & & & 1.70273 \\
\hline 70.2973 & & & 1.67766 \\
\hline 71.9686 & & & 1.65289 \\
\hline 73.6796 & & & 1.62766 \\
\hline 75.0000 & & 6.94110 & \\
\hline 75.4312 & & & 1.60259 \\
\hline 77.2245 & & & 1.57930 \\
\hline 79.0604 & & & 1.55548 \\
\hline 80.0000 & & 6.69725 & \\
\hline 80.9400 & & & 1.53276 \\
\hline 81.1989 & 3.74917 & & \\
\hline 82.8643 & & & 1.51023 \\
\hline 84.8343 & & & 1.48669 \\
\hline 85.0000 & & 6.41516 & \\
\hline 86.8511 & & & 1.46334 \\
\hline 88.9159 & & & 1.43798 \\
\hline 90.0000 & & 6.15326 & \\
\hline 91.0298 & & & 1.41139 \\
\hline 93.1940 & & & 1.38504 \\
\hline 95.0000 & & 5.91041 & \\
\hline 95.4095 & & & 1.35840 \\
\hline 97.6778 & & & 1.33570 \\
\hline 100.000 & & 5.68489 & 1.31354 \\
\hline 110.000 & 2.91709 & 5.34205 & 1.21735 \\
\hline 111.000 & 2.89805 & 5.30567 & 1.20855 \\
\hline 111.200 & 2.89429 & 5.29845 & 1.20679 \\
\hline 111.400 & 2.89052 & 5.29126 & 1.20506 \\
\hline 111.490 & 2.88884 & 5.28803 & 1.20427 \\
\hline 111.518 & 2.85017 & 5.27467 & 1.30362 \\
\hline 111.520 & 2.85012 & 5.27453 & 1.30422 \\
\hline 111.530 & 2.84994 & 5.27418 & 1.30414 \\
\hline 111.540 & 2.84975 & 5.27382 & 1.30405 \\
\hline 111.550 & 2.84956 & 5.27346 & 1.30396 \\
\hline 111.600 & 2.84864 & 5.27168 & 1.30353 \\
\hline 111.800 & 2.84493 & 5.26454 & 1.30179 \\
\hline 112.000 & 2.84123 & 5.25742 & 1.30007 \\
\hline 113.000 & 2.82288 & 5.22214 & 1.29149 \\
\hline 114.000 & 2.80481 & 5.18732 & 1.28300 \\
\hline 115.000 & 2.78706 & 5.15293 & 1.27467 \\
\hline 116.000 & 2.76947 & 5.11906 & 1.26643 \\
\hline 117.000 & 2.75215 & 5.08559 & 1.25830 \\
\hline 118.000 & 2.73505 & 5.05260 & 1.25026 \\
\hline 119.000 & 2.71819 & 5.02004 & 1.24234 \\
\hline
\end{tabular}


TABLE 2. Our recommended elastic ICSs, summed electronic-state excitation ICSs, and the TICSs for electronBe scattering (all in units of $10^{-16} \mathrm{~cm}^{2}$ ) on a fine energy grid. The uncertainties on the elastic ICS are $\sim \pm 10 \%$, the uncertainties on the summed electronic state excitation ICS are $\sim \pm 30 \%$, and the uncertainties on the TICS are $\sim \pm 20 \%$-Continued

\begin{tabular}{|c|c|c|c|}
\hline Energy $(\mathrm{eV})$ & Elastic $\left(\times 10^{-16} \mathrm{~cm}^{2}\right)$ & Inelastic $\left(\times 10^{-16} \mathrm{~cm}^{2}\right)$ & TICS $\left(\times 10^{-16} \mathrm{~cm}^{2}\right)$ \\
\hline 120.000 & 2.70154 & 4.98786 & 1.23451 \\
\hline 122.000 & 2.66889 & 4.92472 & 1.21915 \\
\hline 124.000 & 2.63707 & 4.86316 & 1.20414 \\
\hline 126.000 & 2.60604 & 4.80310 & 1.18951 \\
\hline 128.000 & 2.57578 & 4.74445 & 1.17521 \\
\hline 130.000 & 2.54626 & 4.68685 & 1.16176 \\
\hline 140.000 & 2.40872 & 4.41944 & 1.09702 \\
\hline 150.000 & 2.28609 & 4.17925 & 1.03992 \\
\hline 160.000 & 2.17607 & 4.03246 & 0.989120 \\
\hline 170.000 & 2.07668 & 3.84039 & 0.942510 \\
\hline 180.000 & 1.98629 & 3.66402 & 0.900480 \\
\hline 190.000 & 1.90362 & 3.50121 & 0.862461 \\
\hline 200.000 & 1.82764 & 3.35021 & 0.827554 \\
\hline 225.000 & 1.66221 & 3.08946 & 0.752316 \\
\hline 250.000 & 1.52480 & 2.81128 & 0.689823 \\
\hline 275.000 & 1.40865 & 2.57087 & 0.637316 \\
\hline 300.000 & 1.30901 & 2.36134 & 0.592325 \\
\hline 325.000 & 1.22260 & 2.17750 & 0.553594 \\
\hline 350.000 & 1.14702 & 2.01531 & 0.519497 \\
\hline 375.000 & 1.08029 & 1.87149 & 0.489517 \\
\hline 400.000 & 1.02089 & 1.74338 & 0.462748 \\
\hline 425.000 & 0.967689 & 1.62880 & 0.438834 \\
\hline 450.000 & 0.919796 & 1.52590 & 0.417232 \\
\hline 475.000 & 0.876453 & 1.43313 & 0.397622 \\
\hline 500.000 & 0.837006 & 1.34923 & 0.379836 \\
\hline 550.000 & 0.767909 & 1.26160 & 0.349865 \\
\hline 600.000 & 0.709378 & 1.13702 & 0.323363 \\
\hline 650.000 & 0.659155 & 1.03157 & 0.300578 \\
\hline 700.000 & 0.615573 & 0.941440 & 0.280737 \\
\hline 750.000 & 0.577418 & 0.863749 & 0.263270 \\
\hline 800.000 & 0.543718 & 0.796242 & 0.247805 \\
\hline 850.000 & 0.513749 & 0.737115 & 0.233975 \\
\hline 900.000 & 0.486914 & 0.685023 & 0.221534 \\
\hline 950.000 & 0.462750 & 0.638844 & 0.210337 \\
\hline 1000.00 & 0.440995 & 0.597702 & 0.200092 \\
\hline 1500.00 & 0.299180 & 0.306558 & 0.130937 \\
\hline 2000.00 & 0.226469 & 0.203726 & $9.542578 \times 10^{-2}$ \\
\hline 2500.00 & 0.182212 & 0.147086 & $7.338803 \times 10^{-2}$ \\
\hline 3000.00 & 0.152424 & 0.111960 & $5.843829 \times 10^{-2}$ \\
\hline 3500.00 & 0.131010 & $8.841623 \times 10^{-2}$ & $4.772179 \times 10^{-2}$ \\
\hline 4000.00 & 0.114864 & $7.173270 \times 10^{-2}$ & $3.971776 \times 10^{-2}$ \\
\hline 4500.00 & 0.102252 & $5.942296 \times 10^{-2}$ & $3.357244 \times 10^{-2}$ \\
\hline 5000.00 & $9.212907 \times 10^{-2}$ & $5.004412 \times 10^{-2}$ & $2.873446 \times 10^{-2}$ \\
\hline
\end{tabular}

Finally, in Fig. 2, we compare the present ROP and BEB results for the TICS to the other available theories. ${ }^{2-8}$ From our discussion above, we observed that both the BSR and CCC computations (as well as our ROP result) did a pretty good job in describing both the elastic scattering and summed electronic-state excitation processes. As a consequence, we shall frame the discussion that follows in relation to those theories. The present BEB result predicts a cross section magnitude that is some 20\% higher than the CCC and BSR results (see Fig. 2), and the position in energy of that maximum is shifted somewhat higher compared to those other theories. Indeed the level of accord between our BEB results and the CCC and $\mathrm{BSR}^{7}$ calculations is really quite marginal and quite a bit worse than what is usually found using the $\mathrm{BEB}$ method even though it is only a model (rather than ab initio) approach. ${ }^{10}$ The valence electronic structure of Be can we written as [core] $2 \mathrm{~s}^{2}$, while that of magnesium $(\mathrm{Mg})$ is [core $] 3 \mathrm{~s}^{2}$. Namely, they are both similar in that they possess a pair of s-electrons outside a core. It is well known from electron momentum spectroscopy ${ }^{33}$ that the initial state configuration plays an important role in describing the $\mathrm{Mg} 3 \mathrm{~s}$ momentum distribution. ${ }^{34}$ Specifically, the $3 \mathrm{~s}$ orbital is in fact not a pure "s-type" so that an s/p admixture is required to correctly reproduce the measured $3 \mathrm{~s}$ momentum distribution. ${ }^{34}$ If a similar effect were also occurring in Be, then our BEB result, which employs a pure $2 s^{2}$ ground-state orbital, would be in error. This in turn might explain in part why it (and indeed the model DM result ${ }^{2}$ ) does not reproduce the TICS in 
Fig. 2 to the level we would normally expect. Note that a priori ${ }^{10}$ we would not expect a PWBA result, ${ }^{3,4}$ except at very high energies, to describe well the ionisation process and this is precisely what we find in Fig. 2. The OP TICS from Blanco et al. ${ }^{8}$ predicts a peak maximum which is some $40 \%$ higher in magnitude than those of the BSR and $\mathrm{CCC}$ results ${ }^{7}$ and at an energy that is a little higher than that of the CCC and BSR. These discrepancies are in part addressed by the present ROP calculation. In particular, the energy at which the TICS maximum value occurs, from our ROP computation, is now in excellent agreement with those from the CCC, BSR, DWIS $(\mathrm{N}-1),{ }^{5}$ and RMPS $^{6}$ results. While the magnitude of the TICS maximum from our ROP result is still $\sim 30 \%$ higher than that from the BSR or CCC calculation, this represents an improvement compared to that found by Blanco et al. ${ }^{8}$ with their OP method. We believe this improvement reflects the superior description for exchange and polarisation in our ROP method, compared to their corresponding forms in the work of Blanco et al. ${ }^{8}$ Another interesting aspect in Fig. 2 is the apparent "discontinuity" in the ROP TICS at around an energy of $110 \mathrm{eV}$. This "discontinuity" is in fact physical, corresponding to the opening of the 1s core ionisation channel.

If we consider Fig. 2 in more detail, then four of the $a b$ initio TICS calculations (CC, CCC, RMPS, and BSR) are bunched together with a peak maximum $\sim 2 \times 10^{-16} \mathrm{~cm}^{2}$, while the remaining two ab initio results (ROP, DWIS(N-1)) are bunched with a peak maximum $\sim 3 \times 10^{-16} \mathrm{~cm}^{2}$. In terms of constructing a recommended TICS data base from these results and without any a priori prejudice as to the validity of one method over any other, in the energy range from threshold to $100 \mathrm{eV}$, we have simply taken the average of the CC, CCC, BSR, RMPS, DWIS(N-1) and ROP results. While it could be argued that the track records of the CCC and BSR approaches, in electron-atom scattering and for a range of scattering processes,${ }^{35}$ is such that some preference might be afforded to them, here we have declined to do so. Our rationale for this is that in those cases where the CCC and BSR methods have been shown to be highly successful, at least for a subset of the open scattering channels, they were able to be benchmarked against independent and accurate measurements. This is not the case here. As a consequence, we adopted the approach of Itikawa (e.g., Refs. 36 and 37) who, in the absence of any reliable experimental measurements, suggested a method that might be paraphrased as follows: reject what is inaccurate and average what remains. In doing so cite confidence limits that cover the recommended (averaged) data and all the theory results used to generate that average. In adopting the Itikawa approach here to determine a recommended TICS, we make the following observations. Of the six theories (below $100 \mathrm{eV}$ ) we employed to generate our average, four (CC, CCC, BSR, and RMPS) were bunched quite closely together with the remaining two [DWIS(N-1) and ROP] also being bunched together but at a somewhat higher magnitude (see Fig. 2). Thus in taking the average, the CC, CCC, BSR, and RMPS TICS results will be weighted more strongly than those from the DWIS(N-1) and ROP methods, in forming our recommended TICS data. In addition, with the uncertainty we cite on our recommended TICS (see Sec. III), all those theory

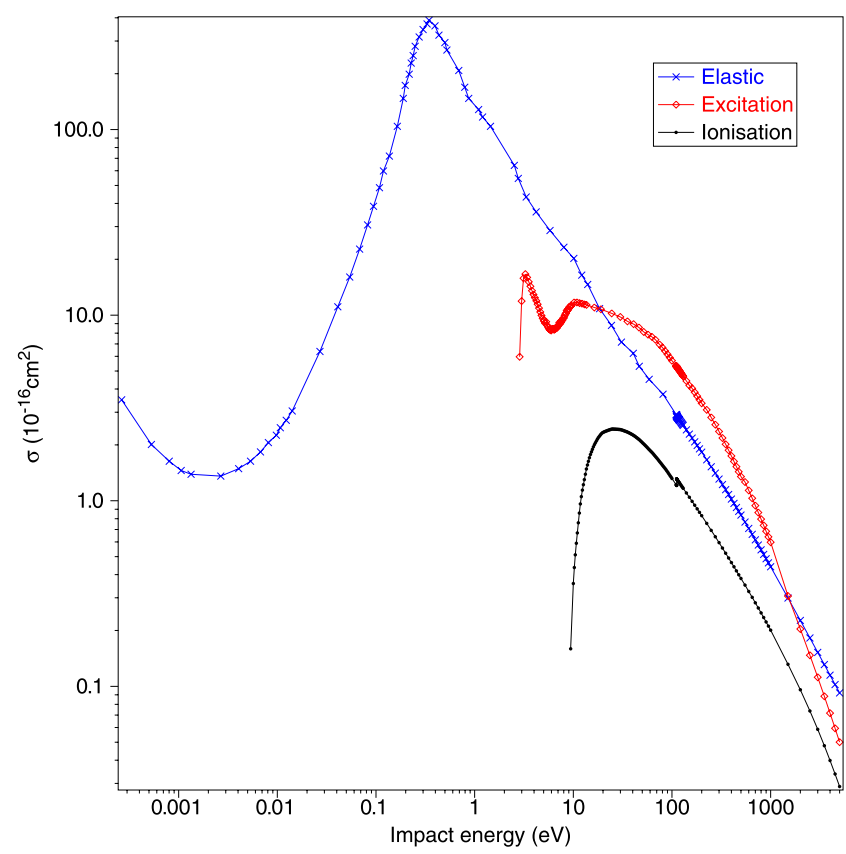

FIG. 3. Summary plot of our recommended cross section data $\left(\times 10^{-16} \mathrm{~cm}^{2}\right)$ for $e^{-}-\mathrm{Be}$ scattering. See also the legend.

results, including the $\mathrm{CCC}$ and BSR, are covered within those confidence limits about our recommended data. The uncertainty on that recommended data is then determined as the standard deviation calculated in taking that average. As the qualitative shapes of the TICS for all the CC, CCC, BSR, RMPS, DWIS(N-1), ${ }^{3-7}$ and ROP calculations are similar, we have used the form of the ROP result, suitably scaled $(\times 0.889)$ to ensure continuity with the averaged data and to extrapolate the TICS from $100 \mathrm{eV}$ to $5000 \mathrm{eV}$. A selection of the results of this process is also presented in Table 2, as are our confidence limits $( \pm 20 \%)$ for this recommended TICS.

In Fig. 3, we therefore provide a summary plot of our recommended cross sections for electron-Be scattering. A listing of all these data, on a fine energy grid, can also be found in Table 2. Note that a fine energy grid is crucial here, to enable modellers to perform an accurate numerical interpolation in order to extract the cross section data at the energies they require to undertake their simulation. This figure nicely illustrates the challenge faced by theory in describing this scattering system, where the magnitude of the ICSs can vary over several orders of magnitude in the energy range considered.

\section{Conclusions}

We have re-examined the available elastic ICSs, summed electronic-state excitation cross sections, and TICSs for $e^{-}-$Be scattering. In order to shed more light on some of the discrepancies between the existing theoretical data, ${ }^{2-8}$ we also undertook new ROP calculations over the $0-5000 \mathrm{eV}$ energy range. As a result of these new computations, recommended cross section data for the above processes over a wide energy range and with confidence limits have also been determined as part of this study. We believe these recommended data will be very useful for modellers seeking to 
better understand the possible action of the ITER plasma on its Be construction components. Finally, we highlight that by summing the recommended elastic ICS, summed electronicstate excitation ICS and TICS, at each energy, of Table 2 then a recommended total cross section for $e^{-}-$Be scattering can also be derived. In practice, however, this would be undertaken after a numerical interpolation of the listed data, in order to extract all those cross sections at each common energy to perform that summation.

\section{Acknowledgments}

This work was financially supported, in part, by the Spanish Ministerio de Economia y Competitividad (Project No. FIS2016-80440) and the Australian Research Council (Project Nos. DP160102787 and DP180101655). We thank Dr. L. Campbell and Dr. D. Jones for their help with some aspects of this study, while one of us (M.J.B.) thanks CSIC for its hospitality during a recent visit. Finally, we also thank Dr. O. Zatsarinny and Professor K. Bartschat for providing us tables of their BSR data.

\section{References}

${ }^{1}$ G. Federici, Phys. Scr. T124, 1 (2006).

${ }^{2}$ T. Maihom, I. Sukuba, R. Janev, K. Becker, T. Märk, A. Kaiser, J. Limtrakul, J. Urban, P. Mach, and M. Probst, Eur. Phys. J. D 67, 2 (2013).

${ }^{3}$ H. Deutsch, P. Scheier, S. Matt-Leubner, K. Becker, and T. D. Märk, Int. J. Mass Spectrom. 243, 215 (2005).

${ }^{4}$ H. Deutsch, P. Scheier, S. Matt-Leubner, K. Becker, and T. D. Märk, Int. J. Mass Spectrom. 246, 113 (2005).

${ }^{5}$ P. L. Bartlett and A. T. Stelbovics, At. Data Nucl. Data Tables 86, 235 (2004).

${ }^{6}$ V. G. Zakrzewski and J. V. Ortiz, J. Phys. Chem. 100, 13979 (1996).

${ }^{7}$ O. Zatsarinny, K. Bartschat, D. V. Fursa, and I. Bray, J. Phys. B: At., Mol. Opt. Phys. 49, 235701 (2016).

${ }^{8}$ F. Blanco, F. Ferreira da Silva, P. Limão-Vieira, and G. García, Plasma Sources Sci. Technol. 26, 085004 (2017).

${ }^{9}$ S. Chen, R. P. McEachran, and A. D. Stauffer, J. Phys. B: At., Mol. Opt. Phys. 41, 025201 (2008).

${ }^{10}$ H. Tanaka, M. J. Brunger, L. Campbell, H. Kato, M. Hoshino, and A. R. P. Rau, Rev. Mod. Phys. 88, 025004 (2016).

${ }^{11}$ M. Vos, R. P. McEachran, C. Cooper, and A. P. Hitchcock, Phys. Rev. A 83, 022707 (2011).

${ }^{12}$ R. P. McEachran, A. D. Stauffer, M. Piwinski, L. Previca, J. F. Williams, D. Cvejanovic, and S. N. Samarm, J. Phys. B: At., Mol. Opt. Phys. 43, 215208 (2010).
${ }^{13}$ C. Makochekanwa, J. R. Mackacek, A. C. L. Jones, P. Caradonna, D. S. Slaughter, R. P. McEachran, S. J. Buckman, B. Lohmann, D. F. Fursa, I. Bray, D. W. Meuller, A. D. Stauffer, and M. Hoshino, Phys. Rev. A 83, 032721 (2011).

${ }^{14}$ R. P. McEachran and A. D. Stauffer, J. Phys. B: At., Mol. Opt. Phys. 46, 075203 (2013).

${ }^{15}$ R. P. McEachran, D. L. Morgan, A. G. Ryman, and A. D. Stauffer, J. Phys. B: At. Mol. Phys. 10, 663 (1977).

${ }^{16}$ R. P. McEachran, D. L. Morgan, A. G. Ryman, and A. D. Stauffer, J. Phys. B: At. Mol. Phys. 11, 951 (1978).

${ }^{17}$ R. P. McEachran and A. D. Stauffer, J. Phys. B: At., Mol. Opt. Phys. 23, 4605 (1990).

${ }^{18}$ I. P. Grant, B. J. McKenzie, P. H. Norrington, D. F. Mayer, and N. C. Pyper, Comput. Phys. Commun. 21, 207 (1980).

${ }^{19}$ A. Kramida and W. C. Martin, J. Phys. Chem. Ref. Data 26, 1185 (1997).

${ }^{20}$ M. J. Frisch et al., GAUSSIAN 09, Revision B.01, Gaussian, Inc., Wallingford, CT, USA, 2010.

${ }^{21}$ R. D. White, W. Tattersall, G. Boyle, R. E. Robson, S. Dujko, Z. Lj. Petrovic, A. Bankovic, M. J. Brunger, J. P. Sullivan, S. J. Buckman, and G. Garcia, Appl. Radiat. Isotopes 83, 77 (2014).

${ }^{22}$ S. J. Buckman, M. J. Brunger, and K. Ratnavelu, Fusion Sci. Technol. 63, 385 (2013).

${ }^{23}$ K. L. Nixon, W. A. D. Pires, R. F. C. Neves, H. V. Duque, D. B. Jones, M. J. Brunger, and M. C. A. Lopes, Int. J. Mass Spectrom. 404, 48 (2016).

${ }^{24}$ W. A. D. Pires, K. L. Nixon, S. Ghosh, R. F. C. Neves, H. V. Duque, R. A. A. Amorim, D. B. Jones, F. Blanco, G. Garcia, M. J. Brunger, and M. C. A. Lopes, Int. J. Mass Spectrom. 422, 32 (2017).

${ }^{25}$ S. Ghosh, K. L. Nixon, W. A. D. Pires, R. A. A. Amorim, R. F. C. Neves, H. V. Duque, D. G. M. da Silva, D. B. Jones, F. Blanco, G. Garcia, M. J. Brunger, and M. C. A. Lopes, Int. J. Mass Spectrom. 430, 44 (2018).

${ }^{26}$ R. D. White, D. Cocks, G. Boyle, M. Casey, N. Garland, D. Konovalov, B. Philippa, P. Stokes, J. de Urquijo, O. González-Magaña, R. P. McEachran, S. J. Buckman, M. J. Brunger, G. Garcia, S. Dujko, and Z. Lj. Petrovic, Plasma Sources Sci. Technol. 27, 053001 (2018).

${ }^{27}$ M. J. E. Casey, J. de Urquijo, L. N. Serkovu Loli, D. G. Cocks, G. J. Boyle, D. B. Jones, M. J. Brunger, and R. D. White, J. Chem. Phys. 147, 195103 (2017).

${ }^{28}$ G. J. Boyle, R. P. McEachran, D. G. Cocks, M. J. Brunger, S. J. Buckman, S. Dujko, and R. D. White, J. Phys. D: Appl. Phys. 49, 355201 (2016).

${ }^{29}$ M. J. Brunger, Int. Rev. Phys. Chem. 36, 333 (2017).

${ }^{30}$ S. T. Perkins, D. E. Cullen, and S. M. Saltzer, Report No. 31, Lawrence Livermore National Laboratory, 1991.

${ }^{31}$ O. Zatsarinny, K. Bartschat, G. Garcia, F. Blanco, L. R. Hargreaves, D. B. Jones, R. Murrie, J. R. Brunton, M. J. Brunger, M. Hoshino, and S. J. Buckman, Phys. Rev. A 83, 042702 (2011).

${ }^{32} \mathrm{~K}$. Bartschat (private communication, 2018).

${ }^{33}$ W. Adcock and M. J. Brunger, J. Chem. Soc., Perkin Trans. 2 2002, 1.

${ }^{34}$ R. Pascual, J. Mitroy, L. Frost, and E. Weigold, J. Phys. B: At., Mol. Opt. Phys. 21, 4239 (1988)

${ }^{35}$ K. Bartschat, J. Phys. B: At., Mol. Opt. Phys. 51, 132001 (2018).

${ }^{36}$ Y. Itikawa, J. Phys. Chem. Ref. Data 44, 013105 (2015).

${ }^{37}$ Y. Itikawa, J. Phys. Chem. Ref. Data 45, 033106 (2016). 